\title{
FSBC: fast string-based clustering for HT-SELEX data
}

Shintaro Kato ${ }^{1,2^{*}}$ (D), Takayoshi Ono ${ }^{2}$, Hirotaka Minagawa ${ }^{1}$, Katsunori Horii ${ }^{1}$, Ikuo Shiratori ${ }^{1}$, Iwao Waga ${ }^{1}$, Koichi Ito ${ }^{2}$ and Takafumi Aoki ${ }^{2}$

\begin{abstract}
Background: The combination of systematic evolution of ligands by exponential enrichment (SELEX) and deep sequencing is termed high-throughput (HT)-SELEX, which enables searching aptamer candidates from a massive amount of oligonucleotide sequences. A clustering method is an important procedure to identify sequence groups including aptamer candidates for evaluation with experimental analysis. In general, aptamer includes a specific target binding region, which is necessary for binding to the target molecules. The length of the target binding region varies depending on the target molecules and/or binding styles. Currently available clustering methods for HT-SELEX only estimate clusters based on the similarity of full-length sequences or limited length of motifs as target binding regions. Hence, a clustering method considering the target binding region with different lengths is required. Moreover, to handle such huge data and to save sequencing cost, a clustering method with fast calculation from a single round of HT-SELEX data, not multiple rounds, is also preferred.

Results: We developed fast string-based clustering (FSBC) for HT-SELEX data. FSBC was designed to estimate clusters by searching various lengths of over-represented strings as target binding regions. FSBC was also designed for fast calculation with search space reduction from a single round, typically the final round, of HT-SELEX data considering imbalanced nucleobases of the aptamer selection process. The calculation time and clustering accuracy of FSBC were compared with those of four conventional clustering methods, FASTAptamer, AptaCluster, APTANI, and AptaTRACE, using HT-SELEX data (>15 million oligonucleotide sequences). FSBC, AptaCluster, and AptaTRACE could complete the clustering for all sequence data, and FSBC and AptaTRACE performed higher clustering accuracy. FSBC showed the highest clustering accuracy and had the second fastest calculation speed among all methods compared.
\end{abstract}

Conclusion: FSBC is applicable to a large HT-SELEX dataset, which can facilitate the accurate identification of groups including aptamer candidates.

Availability of data and materials: FSBC is available at http://www.aoki.ecei.tohoku.ac.jp/fsbc/.

Keywords: SELEX, Aptamer, Next-generation sequencing, Sequence analysis

*Correspondence: katou-s-mxn@nec.com

${ }^{1}$ NEC Solution Innovators, Ltd, 1-18-7 Shinkiba, Koto-ku, 136-8627, Tokyo, Japan

${ }^{2}$ Graduate School of Information Sciences, Tohoku University, 6-6-05 Aramaki

Aza Aoba, Aoba-ku, Sendai-shi, 980-8579, Miyagi, Japan

(C) The Author(s). 2020 Open Access This article is licensed under a Creative Commons Attribution 4.0 International License, which permits use, sharing, adaptation, distribution and reproduction in any medium or format, as long as you give appropriate credit to the original author(s) and the source, provide a link to the Creative Commons licence, and indicate if changes were made. The images or other third party material in this article are included in the article's Creative Commons licence, unless indicated otherwise in a credit line to the material. If material is not included in the article's Creative Commons licence and your intended use is not permitted by statutory regulation or exceeds the permitted use, you will need to obtain permission directly from the copyright holder. To view a copy of this licence, visit http://creativecommons.org/licenses/by/4.0/. The Creative Commons Public Domain Dedication waiver (http://creativecommons.org/publicdomain/zero/1.0/) applies to the data made available in this article, unless otherwise stated in a credit line to the data. 


\section{Background}

Systematic evolution of ligands by exponential enrichment (SELEX) is an experimental method for identifying aptamers, which bind to specific target molecules with high affinity and specificity $[1,2]$. SELEX is an iterative method with multiple rounds for the enrichment of aptamers from the initial oligonucleotide random library. Each round consists of selection with target molecules and amplification with polymerase chain reaction(PCR). Aptamers are RNA or short single-stranded DNA molecules, which fold into a three-dimensional structure and bind different types of target molecules such as proteins [3], small molecules [4], toxins [5], ions [6], and cell surfaces [7]. Owing to the wide variety of possible target molecules, aptamers are commonly used for therapeutics [8], clinical diagnostics [9], the high-throughput multi-protein measurement [10], imaging [11], and biosensors [12].

A next-generation sequencing (NGS), which was originally developed for whole-genome sequencing, is available for analysis of large oligonucleotide pools obtained by SELEX to acquire an enormous sequence dataset for predicting aptamer candidates. This combined use of SELEX and NGS is referred to as high-throughput SELEX (HT-SELEX). It is not reasonable to evaluate the binding affinity with all observed sequences from NGS. In general, dozens of candidate aptamers are selected from the HT-SELEX data for evaluation with experimental analysis considering cost and time-consuming. In other words, the list of dozens of candidate aptamers is required from HT-SELEX data for evaluation with experimental analysis. Clustering for HT-SELEX data is an effective process to identify the sequence groups which are related to aptamer candidates, or noise sequences such as non-specific binding sequences, bead-binders, and PCR biased sequences which are easy to be enriched by PCR. Clustering is also useful to identify different types of aptamers such as different binding epitopes and for understanding the diversity and enrichment of oligonucleotide sequence pools. Figure 1 describes the typical procedure of selecting different types of aptamer candidates from the clustering results for binding verification with experimental analysis.

Several clustering methods have been developed for HT-SELEX data to date, including FASTAptamer [13], AptaCluster [14, 15], APTANI [16], and AptaTRACE [17]. FASTAptamer generates clusters based on Levenshteindistance (LD) which represents the full length of sequence similarity with highly ranked sequences. AptaCluster first roughly groups sequences with local sensitive hashing $(\mathrm{LSH})$ and then generates clusters with the short $k$-mer sequence similarity. APTANI and AptaTRACE identify clusters with short motifs considering the nucleic acid secondary structure. APTANI estimates motifs from a single round of SELEX data whereas AptaTRACE estimates motifs by tracing the changes of frequency between multiple rounds.

It is often observed that the most enriched sequence does not show the binding affinity to the target molecules. These noise sequences are likely to be generated by PCR bias (some oligonucleotide molecules are easy to be enriched by PCR) or non-specific binding of other molecules such as beads with charge effect. Typically, aptamers harbor a specific sequence region, which is necessary for binding to the target molecules, although noise oligonucleotide sequences generally do not include such a target binding region. Hence, determining the sequence clusters with such a target binding region could be an effective approach to choose aptamer candidates. The length of the target binding region varies according to the target molecules, epitopes, and/or binding styles. Thus, estimating target binding regions with different lengths is required. Although AptaTRACE was designed for detecting the candidate motifs as target binding regions, it has a limitation of the length of motifs and requires multiple rounds of SELEX data, which increases the sequencing cost.

To overcome these limitations, we developed the fast string-based clustering (FSBC) method. FSBC estimates clusters considering different lengths of over-represented strings as target binding regions. FSBC was also designed for fast calculation with search space reduction of over-represented strings using only a single round of HT-SELEX data, especially in the final round of SELEX, considering the imbalance of nucleobases of the aptamer selection process. FSBC implemented with $\mathrm{R}$ [18] is available at http://www.aoki.ecei.tohoku.ac.jp/fscb/.

\section{Methods}

\section{Overview of the clustering algorithm}

FSBC is composed of two parts: selection of overrepresented strings with different lengths and sequence clustering based on the selected over-represented strings. For over-represented string selection, we propose a new score calculation method that considers the imbalanced ratios of nucleobases due to the selection process of SELEX. Figure 2 shows the outline of the FSBC algorithm.

\section{String score definition}

For a set of nucleobases $\Omega=\{\mathrm{A}, \mathrm{C}, \mathrm{G}, \mathrm{T}(\mathrm{U})\}$, which represents adenine, cytosine, guanine, and thymine/uracil, respectively, the probability of each nucleobase is given as $p_{j},(j \in \Omega)$, the string is $s$, the length of the string is $|s|$, and the number of nucleobases in the string is $n_{s, j}$. The probability of an $L$-mer oligonucleotide including string $s, P_{s, L}$, 


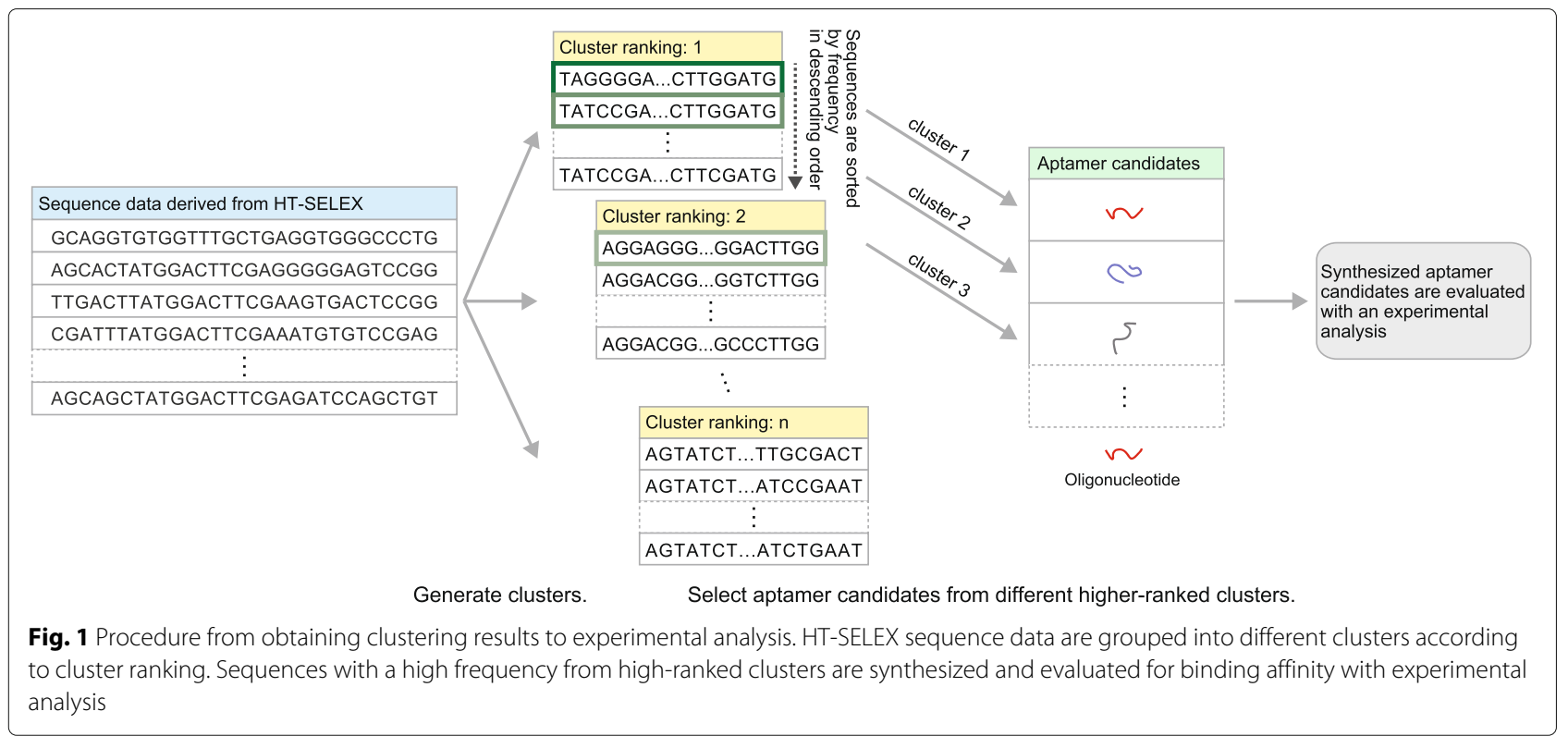

is then described as the following recurrent equation:

$P_{s, L}=P_{s, L-1}+Q\left(1-P_{s, L-|s|}-\sum_{t \in \mathcal{T}} q^{-1}\left(P_{s, L-|s|+|t|}-P_{s, L-|s|+|t|-1}\right)\right)$,

$$
Q=\prod_{j \in \Omega} p_{j}^{n_{s, j}}, \quad q=\prod_{j \in \Omega} p_{j}^{n_{t, j}}, \quad L \geq l
$$

where $\mathcal{T}$ is a set of self-overlapping regions of $s$, and $n_{t, j},(t \in \mathcal{T})$ is the number of nucleobases of the selfoverlapping regions. For example, if string $s$ is "ATATA", the set of self-overlapping regions $\mathcal{T}_{\text {ATATA }}$ is $\{\mathrm{A}, \mathrm{ATA}\}$. If $L<|s|$, then $P_{s, L}=0$. The terms $P_{s, L-1}, Q, Q P_{s, L-|s|}$, and $Q \sum_{t \in \mathcal{T}} q^{-1}\left(P_{s, L-|s|+|t|}-P_{s, L-|s|+|t|-1}\right)$ represent the probability that a sequence has the string from 1 to $L-$ $|s|-1$, a sequence has the string at $L-|s|$, a sequence has the string both from 1 to $L-|s|-1$ and at $L-|s|$, and

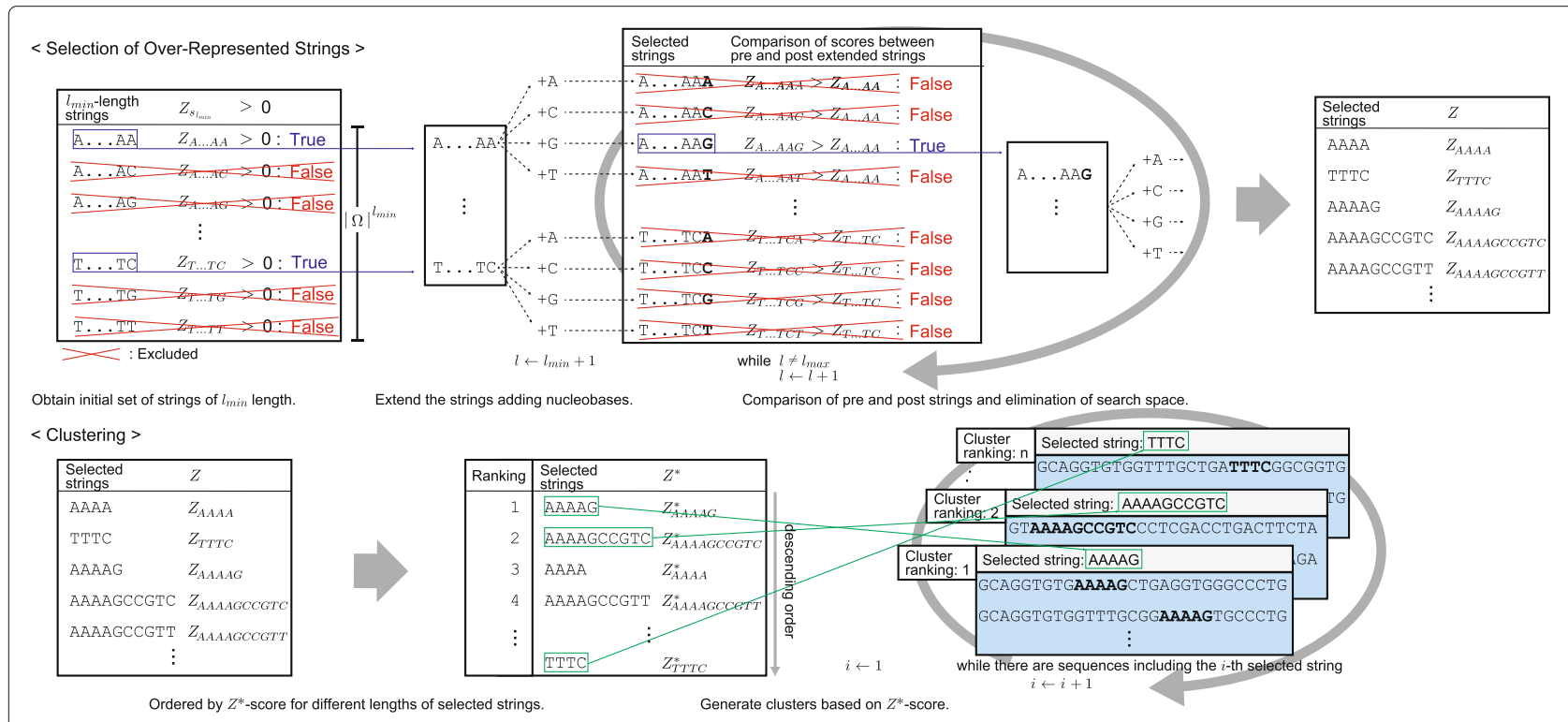

Fig. 2 Outline of fast string-based clustering (FSBC). The algorithm includes over-represented string selection and clustering based on selected strings. The upper panel shows the selection of over-represented strings after minimizing the search space and comparing string scores ( $Z$-scores) of pre- and post- extended strings. The lower panel shows the clustering based on selected strings ranked according to the $Z^{*}$-score, which is normalized Z-score for strings of different lengths 
a sequence has the string at the self-overlapping position, respectively. Figure S1 shows a graphical representation of Eq. (1). In stringology, the probability calculation is the same approach with "missing words in random text" [19], and the self-overlapping region is the same meaning with "string overlaps" [20].

The lengths of observed sequences obtained using NGS vary owing to insertions and/or deletions during the SELEX process. Stoltenburg and Strehiltz described that around $78 \%$ of sequences had an expected length of random regions, while the other $22 \%$ of sequences are different from the original length of random region [21]. Therefore, the probability $P_{s, L}$ was adjusted for different lengths of sequences using the following equation:

$$
P_{s}=\frac{1}{N} \sum_{i=1}^{N} P_{s, L_{i}},
$$

where $N$ is the number of observed sequences and $L_{i}$ is the length of the $i$-th sequence.

Let the frequency of observed sequences including string $s$ be $F_{s} . P_{s}$ follows a binomial distribution. If $N$ is a large enough number for $F_{s}$, a random variable representing the difference between $F_{s}$ and $P_{s}$ normalized by the standard deviation of the binomial distribution then shows an approximate normal Gaussian distribution. Hence, the $Z$-score for string $s$ is derived according to the following equation:

$$
Z_{s}=\frac{\frac{F_{s}}{N}-P_{s}}{\sqrt{\frac{P_{s}\left(1-P_{s}\right)}{N}}} .
$$

\section{Selection of over-represented strings}

Before selection of the over-represented strings, the probability of each nucleobase, $\hat{p}_{j}$, is estimated with the following equation:

$$
\hat{p}_{j}=\frac{n_{j}}{\sum_{i=1}^{N} L_{i}}, \quad j \in \Omega,
$$

where $n_{j}$ is the number of observed nucleobases. These estimated probabilities are then used for calculation of the $Z$-scores. Since the ratios of nucleobases in the SELEX pool can change owing to the systematic selection bias of SELEX, the $Z$-score is calculated based on the balance of nucleobases using Eqs. (1) - (4).

Over-represented strings with lengths ranging from $l_{\text {min }}$ to $l_{\max }$ are selected while reducing the search space from all possible combinations by comparing $Z$-scores. Selection of over-represented strings is then conducted according to the following process:

1 Enumerate all $l_{\text {min }}$-length strings and calculate their $Z$-scores. Exclude string whose $Z$-scores are less than 0.

2 Substitute $l \leftarrow l_{\text {min }}$.
3 Enumerate extended strings by adding a nucleobase and calculate their $Z$-scores. Exclude extended strings whose $Z$-scores are less than those of the pre-extended strings.

4. If $l+1>l_{\text {max }}$, then finish the selection of over-represented strings.

5 Substitute $l \leftarrow l+1$, and go to 3 .

The algorithm for estimating over-represented strings reduces the search space by comparing of $Z$-scores between the post-extended and pre-extended strings. Thus, the number of selected strings, $m$, is much smaller than the exhaustive enumeration of all strings: $m \ll$ $\sum_{l=l_{\min }}^{l_{\max }}|\Omega|^{l}$. This search space minimization provides a huge reduction in the calculation time for an HT-SELEX dataset.

\section{Clustering with selected over-represented strings}

While extending the string length, the strings with higher Z-scores are selected for search space reduction. For evaluating the different lengths of strings equally, the normalization of the Z-score was performed. The normalized $Z$-score for string $s$, referred to as $Z_{s}^{*}$, is calculated with the following equation:

$$
Z_{s}^{*}=\frac{Z_{s}-\hat{\mu}_{|s|}}{\hat{\sigma}_{|s|}}
$$

where $\hat{\mu}_{|s|}$ and $\hat{\sigma}_{|s|}$ are the mean and standard deviation, respectively, of the $Z$-score of selected strings with length $|s|$. The strings are then ordered by $Z^{*}$. Parameters $\hat{\mu}_{|s|}$ and $\hat{\sigma}_{|s|}$ are estimated with only selected strings. Therefore, there are no guarantees of Gaussian distribution of $Z^{*}$. The clustering is then achieved according to the following process:

1 Substitute $i \leftarrow 1$.

2 Extract sequences including the $i$-th strings from the sequence dataset, where a set of extracted sequences is referred to as the $i$-th cluster. Remove extracted sequences from the sequence dataset.

3 If there are no sequences remaining, finish the clustering.

4. Substitute $i \leftarrow i+1$, and go to step 2 .

\section{Data}

The publicly available whole-cell SELEX dataset of human embryonic stem cells [22] was used for comparing the calculation speed and clustering accuracy. The SELEX was finished at the fifth round and nineteen sequences were evaluated for binding affinity with flow cytometry. According to the binding evaluation, eight of nineteen sequences showed higher fluorescent intensity and those sequences were defined as target-binding sequences. 


\section{Calculation time}

The sequence data were filtered with different frequency cut-offs $(1,10$, and 100) to vary the size of the dataset. The numbers of sequences included with frequency cut-offs of 1,10 , and 100 were $15,327,604(4,381,160), 8,799,219$ $(156,587)$, and $4,947,522(6,193)$ with 1,10 , and 100 , respectively; the numbers of non-redundant sequences are indicated in parentheses.

The five different algorithms, namely FASTAptamer, AptaCluster, APTANI, AptaTRACE, and FSBC, were compared with respect to calculation time. The fifth round HT-SELEX data, which was the last round of SELEX, were used for FASTAptamer, AptaCluster, APTANI, and FSBC. The fourth and fifth round HTSELEX data were used for AptaTRACE because AptaTRACE requires multiple rounds of HT-SELEX data.

FASTAptamer was performed with an edit distance option of 7 (according to the user guide), and the maximum cluster number was set to 100 to reduce the calculation time. AptaCluster was performed with the default options. The options for APTANI were no-filtering of frequency, fixed length for HT-SELEX data, and primer information for estimation of the secondary structure. There are no further options for reducing the calculation time except for frequency filtering; thus, we did not change any options for APTANI. AptaTRACE was performed with the background sequence option as 1,000 because AptaTRACE demonstrated the best accuracy with that parameter. The options of FSBC were $l_{\min }=5$ and $l_{\text {max }}=10$.

FSBC was written in R [18] version 3.6.2 with Bioconductor packages [23], and other programs are provided with scripts and executable files. The computer specifications were as follows: OS Ubuntu 16.04 (Xenial Xerus) 64bit, Intel(R) Xeon(R) CPU E5-1650v4@3.60GHz, and 64 GB memory.

\section{Clustering accuracy}

Filtered data (frequency $\geq 10$ ) of the fifth round, which was the final round of the SELEX, was applied for comparing the accuracy of the clustering methods because
FASTAptamer and APTANI did not complete the clustering with the entire sequence dataset. The same parameters indicated in the previous subsection for AptaCluster and APTANI were applied for evaluating the clustering accuracy. The option of the maximum number of clusters for FASTAptamer was not used for the evaluation of clustering accuracy. Changing the parameters of LD and motif length did not improve the accuracy of FASTAptamer and AptaCluster, respectively. For AptaTRACE, the background sequence option was set as 1,000 because AptaTRACE showed the highest accuracy with that option. The options for FSBC were $l_{\min }=\{3,4,5\}$ and $l_{\max }=$ 10. FSBC was also applied to the entire sequence dataset and the filtered data (frequency $\geq 100$ ) to evaluate the potential bias of frequency filtering, and missing aptamer sequences due to the sequence frequency filtering.

The sequences with binding/non-binding information were sorted with cluster ranking for each method. For evaluating the cluster ranking and binding sequences, the receiver operating characteristic (ROC) curves were generated according to the order of cluster ranking with the binding information. The area under the curve (AUC) values were calculated based on the area of the ROC curves. FSBC was also applied to all of the sequence data from the third and fourth rounds of SELEX to evaluate the possibility of the detection of aptamers in early rounds.

\section{Comparison with exhaustive enumeration of strings}

Due to the search space reduction, there are no guarantees that the top-ranked strings of exhaustive enumeration are included in the selected strings. Hence, we verified whether the top-ranked strings of exhaustive enumeration was included in the selected strings or not. The missing rate of the top ten ranked strings of exhaustive enumeration was also evaluated for each length.

\section{Results}

\section{Calculation time}

Table 1 shows the calculation time for each method and the dataset size. The first column shows the clustering methods, and the second to seventh columns represent

Table 1 Clustering calculation time for each method with datasets of different sizes. Sequences ( $\geq 10)$ and sequences ( $\geq 100)$ represent filtered data with frequency cutoff. DNF indicates did not finish. DNF ${ }^{1}$ : FASTAptamer did not complete the calculation for the entire sequence dataset in 7 days. DNF²: APTANI showed a calculation error after the prediction of the secondary structure, which took $25 \mathrm{~h}$

\begin{tabular}{|c|c|c|c|c|c|c|}
\hline \multirow[b]{2}{*}{ Method } & \multicolumn{2}{|l|}{ All sequences } & \multicolumn{2}{|c|}{ Sequences $(\geq 10)$} & \multicolumn{2}{|c|}{ Sequences $(\geq 100)$} \\
\hline & Real time & CPU time & Real time & CPU time & Real time & CPU time \\
\hline FASTAptamer & $\mathrm{DNF}^{1}$ & $\mathrm{DNF}^{1}$ & $5 \mathrm{~h} 16 \mathrm{~m} \mathrm{4s}$ & $5 \mathrm{~h} 16 \mathrm{m3s}$ & $10 \mathrm{~m} 40 \mathrm{~s}$ & $10 \mathrm{~m} \mathrm{40s}$ \\
\hline AptaCluster & $3 \mathrm{~m} 45 \mathrm{~s}$ & $4 \mathrm{~m} 9 \mathrm{~s}$ & $33 \mathrm{~s}$ & $26 s$ & $28 \mathrm{~s}$ & $17 \mathrm{~s}$ \\
\hline APTANI & $\mathrm{DNF}^{2}$ & $\mathrm{DNF}^{2}$ & $32 \mathrm{~m} 52$ & $34 \mathrm{~m} 59 \mathrm{~s}$ & $1 \mathrm{~m} 47 \mathrm{~s}$ & $1 \mathrm{~m} 20 \mathrm{~s}$ \\
\hline AptaTRACE & 71 h 38 m 35 s & $246 \mathrm{~h} 15 \mathrm{~m} 12 \mathrm{~s}$ & $1 \mathrm{~h} 1 \mathrm{~m} \mathrm{17s}$ & $2 \mathrm{~h} 2 \mathrm{~m} 50 \mathrm{~s}$ & $3 \mathrm{~m} 52 \mathrm{~s}$ & $5 \mathrm{~m} 44 \mathrm{~s}$ \\
\hline FSBC & $4 \mathrm{~h} 40 \mathrm{~m} 51 \mathrm{~s}$ & $4 \mathrm{~h} 40 \mathrm{~m} 34 \mathrm{~s}$ & $9 \mathrm{~m} 25 \mathrm{~s}$ & $9 \mathrm{~m} \mathrm{17s}$ & $51 \mathrm{~s}$ & $46 s$ \\
\hline
\end{tabular}


the actual and CPU time for each size of dataset. Note that the calculation time of FASTAptamer includes the preprocessing time, which involves counting the frequency of sequences, before clustering.

AptaCluster showed the fastest calculation time for clustering, followed by FSBC. However, FASTAptamer was the slowest of the five methods and did not complete the clustering of the entire dataset in 7 days, even when changing the clustering number option to "-c 100 " to reduce the calculation time. APTANI also could not complete the calculation for the entire dataset due to an error after the secondary structure prediction, which required $25 \mathrm{~h}$. AptaTRACE calculated clustering with parallel computing, hence the real-time was much smaller than CPU time.

\section{Accuracy}

The clustering result for each algorithm is shown in Table 2. The columns indicate the oligonucleotide sequences excluding both ends of the primers, sequence ID, ranking of frequency, frequency, binding information, and cluster ranking for each method. AptaCluster has a two-ranking system for clustering, including frequency and diversity, corresponding to the frequency of sequences in the cluster and the number of nonredundant sequences in the cluster, respectively. APTANI does not include any functions for ordering clusters; thus, we used frequency and diversity for this purpose as performed by AptaCluster. The binding information was already defined by the verification of experimental analysis using flow cytometry [22]. Sequence IDs, seq1 to seq 8 are defined as binding sequences whereas sequence IDs seq 9 to seq 19 are not the binding sequences. Sequence ID seq 8 was not included since it was filtered out based on the frequency cut-off before clustering. The strings selected by FSBC are underlined and in uppercase in the table. The order of sequences in Table 2 is based on the ranking of the frequency on binding/nonbinding sequences. FASTAptamer, AptaCluster (frequency/diversity), APTANI (frequency/diversity), AptaTRACE and FSBC estimated 2,380, 136,350, 2,348, 13, and 155 clusters, respectively.

Among the five methods, only FSBC and AptaTRACE provided a top-ranked cluster that included binding sequences. By contrast, the top-ranked clusters obtained with FASTAptamer, AptaCluster (frequency), and APTANI, and the second top-ranked cluster obtained by AptaCluster (diversity) included the topranked sequence of "frequency", which did not show binding ability. Similarly, APTANI (diversity) yielded a topranked cluster including sequence ID seq17, which also did not bind to the target molecules. FASTAptamer, AptaCluster (frequency), AptaCluster (diversity), APTANI (frequency), and APTANI (diversity) showed 6, 7, 5, 7, and 290 as the highest ranked clusters including binding sequences, respectively, and these ranks were all lower than those with non-binding sequences. FSBC and AptaTRACE grouped all binding sequences from sequence ID seq1 to seq7 into two clusters with cluster ranks 1 and 5. However, AptaTRACE missed sequence ID seq6, and sequence ID seq7 was grouped with sequence ID seq17 which did not show the binding affinity. FASTAptamer grouped sequence ID seq2 and seq4 into the same cluster, which was ranked fifteenth. APTANI (diversity) showed the same cluster ranking from sequence ID, seq5 to seq8; however, these ranks were simply tied but the sequences did not group in the same cluster. AptaCluster (frequency/diversity) and APTANI (frequency) did not group any binding sequences into the same cluster. Table $\mathrm{S} 1$ shows the same result of FSBC with all sequences (nofiltering with frequency cutoff) and filtered data $(\geq 100)$ under the option $l_{\min }=5$. Similar to the result in Table 2, all binding sequences were in the higher-ranked clusters rather than in the clusters ranked with non-binding sequences.

FSBC selected a total of 1,003 strings, and the top 24 strings are shown in Table S2. The selected overrepresented strings "ATGGACTTCGG" and "GACTT", ranked 1 and 12, respectively, were included in cluster 1 and 5 in Table 2. The selected string "GACTT" is a part of string "ATGGACTTCGG". The distribution of the $Z$ scores and $Z^{*}$-scores of the selected strings for each length of string is shown in Figure S2.

The relation between cluster ranking and frequency of oligonucleotide sequences with each method is displayed in Fig. 3, in which the red, blue, and gray dots represent binding, non-binding, and non-evaluated sequences to the target molecules, respectively. The top-ranked clusters obtained by FASTAptamer, AptaCluster (frequency), and APTANI (frequency) included the non-binding sequence of the highest frequency. AptaCluster (diversity) and APTANI (diversity) included the non-binding sequence of the highest frequency in higher ranked cluster than those including binding sequences. By contrast, FSBC and AptaTRACE grouped the binding sequences with lower frequencies in the top-ranked cluster.

The ROC curve and AUC value for each clustering method are displayed in Fig. 4. FSBC with options $l_{\min }=4$ and $l_{\min }=5$ clearly distinguished binding from nonbinding sequences, i.e. the AUC value equals to 1 . The AUC value was slightly lower (0.96) when the FSBC options $l_{\min }=3$ were applied, because some non-binding sequences were grouped into the same binding cluster. AptaTRACE also demonstrates a higher AUC value because AptaTRACE detected the target binding regions in the higher-ranked clusters. However, the other clustering methods resulted in lower AUCs because non-binding sequences with high frequency were included in the 


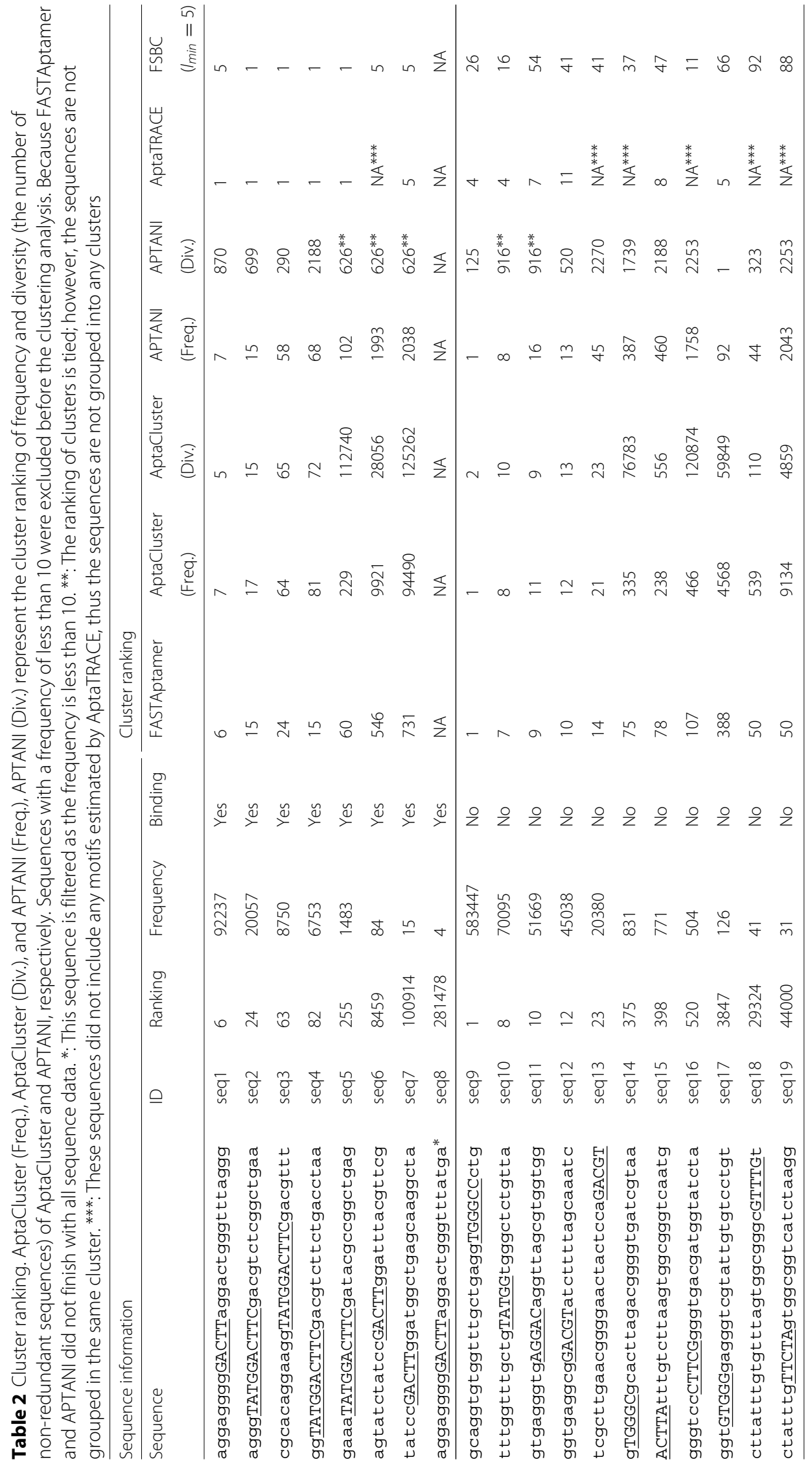




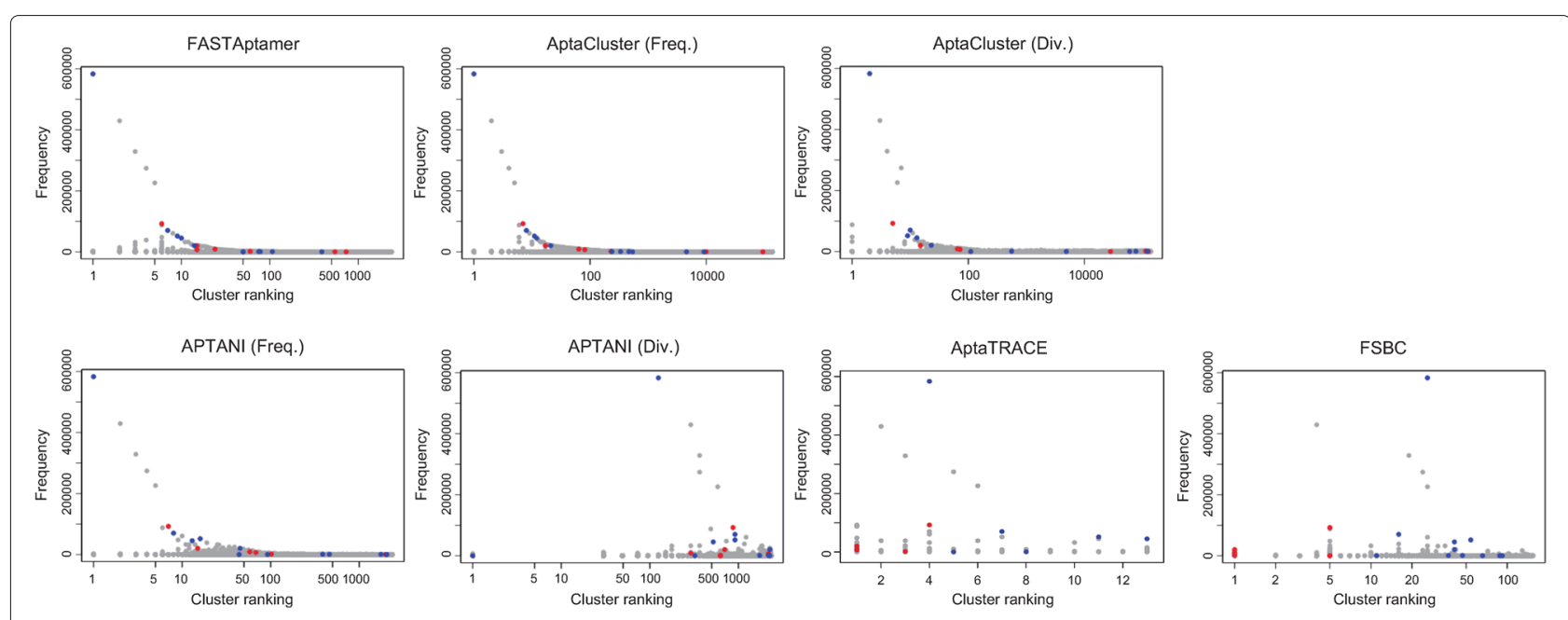

Fig. 3 Relation between cluster ranking and frequency of sequences. Binding, non-binding, and non-evaluated sequences are shown as red, blue, and gray dots, respectively

higher-ranked clusters. FSBC with option $l_{\min }=5$ also showed that the AUC value equals to 1 for all sequence data and filtered data (frequency $\geq 100$ ) in Table S1. The clustering results for third- and fourth-round data are summarized in Table S3 and Table S4, respectively. FSBC could identify aptamer sequences in the third and first clusters from third-and fourth-round data. AUC values for the third and fourth rounds are 0.89 and 1 , respectively.

\section{Comparison with exhaustive enumeration of strings}

The top-ranked over-represented string of exhaustive enumeration was included in the selected strings for each length. The missing rate of the top 10 ranked strings of exhaustive enumeration for each length is shown in Table S5. Top 10 ranked 10-mer strings of the exhaustive enumeration include 6 selected strings. Thus, the missing rate of the 10-mer string is 0.4 .

\section{Discussion}

Our newly developed algorithm for clustering, FSBC, showed the second fastest calculation speed with HTSELEX data. AptaCluster displayed a remarkably fastest calculation time. FASTAptamer and APTANI could not complete the clustering for all of the sequence data; hence, only FSBC, AptaCluster, and AptaTRACE are available for applications with a real HT-SELEX dataset. FSBC selected a total of 1,003 strings, which was much smaller than all exhaustive enumeration of strings: $\sum_{i=5}^{10} 4^{i}=1,397,760$. The ratio of the number of selected strings over all combinations is $1,003 / 1,397,760=0.0007175767$. Hence, the minimization of the search space was an effective method for finding over-represented strings of longer lengths such as 10-mer. FSBC was designed for handling a single-round sequence data from SELEX. This approach could also be helpful to reduce the sequencing cost and the calculation time compared to other methods such as MPBind [22] and AptaTRACE[17], which require multiple rounds of sequence data from SELEX pools.

Importantly, FSBC and AptaTRACE distinguished binding sequences as high-ranked clusters, whereas the other clustering methods categorized non-binding sequences with high frequency under high-ranked clusters. This demonstrates that FASTAptamer, AptaCluster, and APTANI are more sensitive to the frequency of sequences rather than to enrichment of over-represented strings. Thus, if the SELEX pool contains numerous non-binding sequences due to PCR bias, FASTAptamer, AptaCluster, and APTANI might place these PCR biased sequences in the high-ranked clusters. The sequencing data used for the current study includes enriched strings among the binding sequences, and FSBC and AptaTRACE could accurately detect these strings as the estimated target binding region. AptaTRACE detected binding sequences with higher-ranked clusters, however, the cluster of rank 5 includes both binding and non-binding sequences. Consequently, FSBC showed a better result for cluster ranking in this study.

This proposed string score calculation method can be extended to combine with other outcomes. In this study, we defined the outcome according to nucleobases: $\Omega_{\text {nucleobase }}=\{\mathrm{A}, \mathrm{C}, \mathrm{G}, T(U)\}$. However, other outcomes can also be defined, such as the oligonucleotide secondary structure: $\Omega_{\text {structure }}=\{\mathrm{H}, \mathrm{B}, \mathrm{S}, \mathrm{M}, \mathrm{E}, \mathrm{I}, \mathrm{G}\}$, which represent the structure of the hairpin loop, bulge, stem, multi-loop, external loop, internal loop, and G-quadruplex, respectively. A set of outcomes can be extended as $\Omega=$ $\Omega_{\text {nucleobase }} \times \Omega_{\text {structure }}$. If the set is extended to include the secondary structure, FSBC is available for searching 


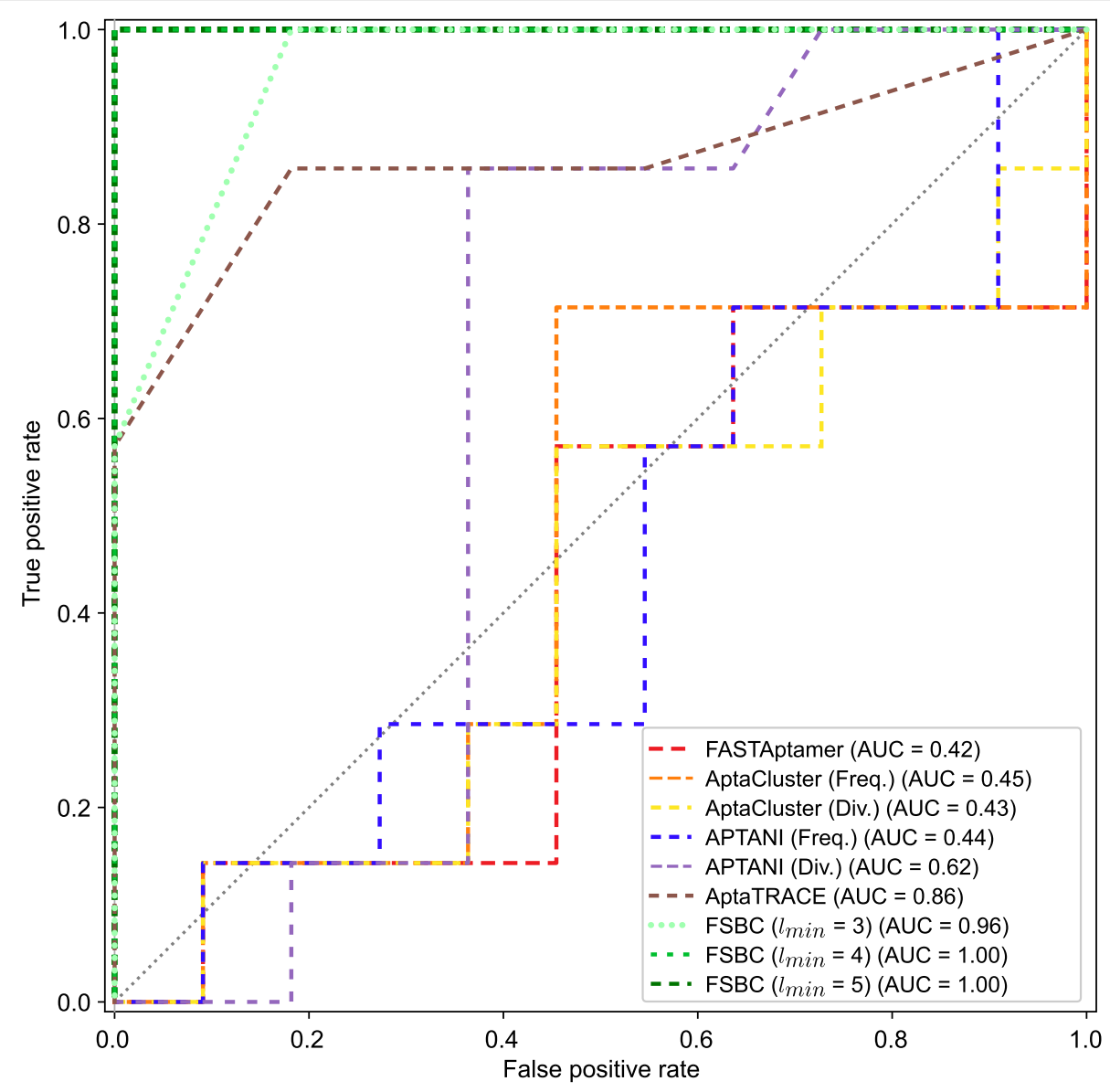

Fig. 4 Receiver operating characteristic (ROC) curves of different clustering methods. "Freq." and "Div." in the parentheses (after AptaCluster and APTANI) indicate the cluster ranking with frequency and diversity (the number of non-redundant sequences) in the cluster for the respective method. AUC indicates the area under the curve

over-represented strings with a specific secondary structure. However, the calculation time will also increase with increasing the number of elements of $\Omega$. Hence, to obtain the fastest calculation with FSBC, $\Omega=\Omega_{\text {nucleobase }}$ is the reasonable outcome. This string scoring method can also be used for other types of sequence analysis such as for amino acid sequences. In other words, if $\Omega$ is defined based on amino acids, Eq. (1) can be used for finding over-represented strings among amino acid sequences.

FSBC does not consider insertion/deletions or degenerated nucleobases, because the method was designed to reduce the calculation time to enable estimating longer over-represented strings in a huge dataset. Since the size of clusters is much smaller than the size of the entire sequence dataset, other motif-estimating methods such as MEME [24] can be used for more accurate estimation of candidate motifs.

Due to a lack of publicly accessible HT-SELEX data with binding information, only one HT-SELEX dataset was used. Sequence data could differ depending on the target molecules, SELEX methods, and initial bias of SELEX. Hence, the evaluation with other HT-SELEX data should be performed. After there will be enough dataset of HTSELEX data publicly available for evaluation, the clustering methods need to be summarized. Moreover, only a single clustering method cannot cover all types of SELEX datasets. Thus, the most suitable clustering approach is to compare and summarize the results of different clustering methods.

\section{Conclusion}

We proposed a new and rapid string-based clustering method for HT-SELEX data. Our clustering method could complete the calculation from a huge dataset in a reasonable time, even though the method is designed to estimate longer over-represented strings such as 10-mer. Importantly, our clustering method could identify enriched strings that were included in binding sequences estimated as the target binding region of the aptamer. Overall, FSBC 
could be a helpful method to effectively identify aptamers with HT-SELEX data.

\section{Supplementary information}

Supplementary information accompanies this paper at https://doi.org/10.1186/s12859-020-03607-1.

Additional file 1: The supplementary document includes supplementary tables (Tables S1 to S5) and figures (Figures S1 and S2).

\section{Abbreviations}

FSBC: Fast string-based clustering; SELEX: Systematic evolution of ligands by exponential enrichment; HT-SELEX: High-throughput systematic evolution of ligands by exponential enrichment; NGS: Next-generation sequencing; LD: Levenshtein-distance; LSH: Locality sensitive hashing; ROC: Receiver operating characteristic; AUC: Area under the curve; PCR: Polymerase chain reaction

\section{Acknowledgements}

The authors are grateful to the researchers of Innovation Laboratories of NEC Solution Innovators and members of Computer Structures Laboratory of Graduate School of Information Sciences, Tohoku University, for their helpful discussions.

\section{Authors' contributions}

SK, TO, HM, KH, IS, IW, KI, and TA conceived and designed the study. SK, TO, KI, and TA developed the method. SK and TO implemented the programs and analyzed the data. SK, TO, and KI drafted the manuscript. All authors have read and approved the manuscript.

\section{Funding}

A part of this work was supported by JSPS KAKENHI Grant Number $18 \mathrm{H} 03253$.

\section{Availability of data and materials}

FSBC was implemented with R version 3.6.2 and is available at http://www. aoki.ecei.tohoku.ac.jp/fsbc/.

\section{Ethics approval and consent to participate}

Not applicable.

\section{Consent for publication}

Not applicable.

\section{Competing interests}

SK, HM, KH, IS, and IW are employees of NEC Solution Innovators, Ltd. The company did not have any additional role in the study design, data collection and analysis, decision to publish, or preparation of the manuscript. All other authors declare that they have no competing interests.

Received: 2 July 2019 Accepted: 16 June 2020

Published online: 24 June 2020

\section{References}

1. Ellington AD, Szostak JW. In vitro selection of rna molecules that bind specific ligands. Nature. 1990;346(6287):818.

2. Tuerk C, Gold L. Systematic evolution of ligands by exponential enrichment: Rna ligands to bacteriophage 44 dna polymerase. Science. 1990;249(4968):505-10.

3. Bock LC, Griffin LC, Latham JA, Vermaas EH, Toole JJ. Selection of single-stranded dna molecules that bind and inhibit human thrombin. Nature. 1992;355(6360):564.

4. Zimmermann GR, WICK CL, SHIELDS TP, JENISON RD, PARDI A. Molecular interactions and metal binding in the theophylline-binding core of an rna aptamer. Rna. 2000;6(5):659-67.

5. Cunha I, Biltes R, Sales M, Vasconcelos V. Aptamer-based biosensors to detect aquatic phycotoxins and cyanotoxins. Sensors. 2018;18(7):2367.

6. Qu H, Csordas AT, Wang J, Oh SS, Eisenstein MS, Soh HT. Rapid and label-free strategy to isolate aptamers for metal ions. ACS nano. 2016;10(8):7558-65
7. Marton S, Cleto F, Krieger MA, Cardoso J. Isolation of an aptamer that binds specifically to e. coli. PLoS ONE. 2016;11(4):0153637.

8. Ng EW, Shima DT, Calias P, Cunningham Jr ET, Guyer DR, Adamis AP. Pegaptanib, a targeted anti-vegf aptamer for ocular vascular disease. Nat Rev Drug Discov. 2006;5(2):123.

9. Ruiz Ciancio D, Vargas M, Thiel W, Bruno M, Giangrande P, Mestre M. Aptamers as diagnostic tools in cancer. Pharmaceuticals. 2018;11(3):86.

10. Gold L, Ayers D, Bertino J, Bock C, Bock A, Brody EN, Carter J, Dalby AB, Eaton BE, Fitzwater T, et al. Aptamer-based multiplexed proteomic technology for biomarker discovery. PloS ONE. 2010;5(12):15004.

11. Röthlisberger $P$, Gasse $C$, Hollenstein M. Nucleic acid aptamers: Emerging applications in medical imaging, nanotechnology, neurosciences, and drug delivery. Int J Mol Sci. 2017;18(11):2430.

12. Kaneko N, Horii K, Akitomi J, Kato S, Shiratori I, Waga I. An aptamer-based biosensor for direct, label-free detection of melamine in raw milk. Sensors. 2018;18(10):3227.

13. Alam KK, Chang JL, Burke DH. Fastaptamer: a bioinformatic toolkit for high-throughput sequence analysis of combinatorial selections. Mol Ther Nucleic Acids. 2015:4:230.

14. Hoinka J, Berezhnoy A, Sauna ZE, Gilboa E, Przytycka TM. AptaCluster - A Method to Cluster HT-SELEX Aptamer Pools and Lessons from its Application. Res Comput Mol Biol. 2014;8394:115-28. https://doi.org/10 1007/978-3-319-05269-4_9.

15. Hoinka J, Berezhnoy A, Dao P, Sauna ZE, Gilboa E, Przytycka TM. Large scale analysis of the mutational landscape in ht-selex improves aptamer discovery. Nucleic Acids Res. 2015;43(12):5699-707.

16. Caroli J, Taccioli C, De La Fuente A, Serafini P, Bicciato S. Aptani: a computational tool to select aptamers through sequence-structure motif analysis of ht-selex data. Bioinformatics. 2015;32(2):161-4.

17. Dao P, Hoinka J, Takahashi M, Zhou J, Ho M, Wang Y, Costa F, Rossi JJ, Backofen R, Burnett J, et al. Aptatrace elucidates rna sequence-structure motifs from selection trends in ht-selex experiments. Cell Syst. 2016;3(1): 62-70.

18. R Core Team. R: A Language and Environment for Statistical Computing Vienna, Austria: R Foundation for Statistical Computing; 2013. http:// www.R-project.org/, R Foundation for Statistical Computing.

19. Rahmann S, Rivals E. On the distribution of the number of missing words in random texts. Comb Probab Comput. 2003;12(1):73-87.

20. Guibas $L$, Odlyzko AM. String overlaps, pattern matching, and nontransitive games. J Comb Theory Ser A. 1981;30(2):183-208.

21. Stoltenburg R, Strehlitz B. Refining the results of a classical selex experiment by expanding the sequence data set of an aptamer pool selected for protein a. Int J Mol Sci. 2018;19(2):642.

22. Jiang P, Meyer S, Hou Z, Propson NE, Soh HT, Thomson JA, Stewart R. Mpbind: a meta-motif-based statistical framework and pipeline to predict binding potential of selex-derived aptamers. Bioinformatics. 2014;30(18): 2665-7.

23. Gentleman RC, Carey VJ, Bates DM, Bolstad B, Dettling M, Dudoit S, Ellis B, Gautier L, Ge Y, Gentry J, et al. Bioconductor: open software development for computational biology and bioinformatics. Genome Biol. 2004;5(10):80.

24. Bailey TL, Williams N, Misleh C, Li WW. Meme: discovering and analyzing dna and protein sequence motifs. Nucleic Acids Res. 2006;34:369-73.

\section{Publisher's Note}

Springer Nature remains neutral with regard to jurisdictional claims in published maps and institutional affiliations. 